\title{
Machine Vision System for Color Sorting Wood Edge-Glued Panel Parts
}

\author{
Q. Lu, S. Srikanteswara, W. King, T. Drayer, R. \\ Conners, E. Kline* \\ The Bradley Department of Electrical and Computer Eng. \\ *Department of Wood Science and Forest Products \\ Virginia Tech, Blacksburg, VA 24061-0111
}

\author{
P. Araman \\ Southern Forest Research Station \\ U.S. Forest Service \\ Blacksburg, VA 24061-0503
}

\begin{abstract}
This paper describes an automatic color sorting system for hardwood edge-glued panel parts. The color sorting system simultaneously examines both faces of a panel part and then determines which face has the "better" color given specified color uniformity and priority defined by management. The real-time color sorting system software and hardware are briefly described. An actual working system has undergone extensive plant testing capable of sorting red-oak panel parts into a number of color classes at plant production speeds. Initial test results show that the system can generate over 91 percent acceptable panels from automatically sorted panel parts. These results exceeded target plant production goals.
\end{abstract}

\section{INTRODUCTION}

Color sorting of edge-glued panel parts is an important manufacturing step where color uniformity has an impact on the value of the final products. If performed manually, proper color sorting of edge-glued panel parts is very labor intensive. Also, different people have different perceptions about color uniformity in hardwood panel products. Therefore, uniformity and consistency in edge-glued panel color is very difficult to achieve at production speeds through manual color sorting.

Because a distinct market preference for color uniformity exists, a number of researchers have examined the color characteristics of wood [1], [2], [6], [10], [13]. These researchers have studied color measurement systems and how they can be applied to automatically inspect wood. While these studies have developed systems to better characterize color in wood, they have not led directly to any commercially available systems that can meet the demands of the wood processing industry. In creating a color sorting system that will work in industry, it must (1) be able to accurately and consistently separate parts into appropriate color classes, (2) be able to keep up with the production requirements of the plant, and (3) be easy to operate by plant production personnel.
The purpose of this paper is to describe a color sorting system for panel parts that was developed at Virginia Tech. A software algorithm for color sorting edge-glued panel parts is first described in this paper. Then, the color imaging and real-time image processing hardware necessary to implement the software at production speeds in the plant are described. Finally, initial results of the in-plant performance for a prototype color sorting system are discussed.

\section{COLOR SORTING ALGORITHM DESCRIPTION}

The key to color sorting panel parts is defining a measurement vector that accurately gauges all the natural color variations in wood. To truly represent the color characteristics of wood, a full three-dimensional (3-D) color histogram was used as the measurement vector for the color characteristics of a part face. 3-D color histograms were needed to overcome color separation problems found with simpler one-dimensional methods of color measurement [13]. Unfortunately, 3-D color histograms are very large. This makes using these histograms to do the color sorting rather computationally complex. To reduce computationally complexity, the techniques used on this color sorting system employ a color mapping algorithm, a modified version of the one described in [7], to reduce the size of the measurement vector while preserving the necessary 3-D color information. It has been experimentally determined that when this mapping is used a 3-D color histogram for red oak panel parts can be accurately represented using 2000 color elements. This significantly reduced measurement vector still has the capability to overcome color separation problems encountered in previous research. For example, a full $64 \times 64 \times 643-D$ color histogram with over 260,000 elements can be reduced to a measurement vector that is only 2000 elements long. The color mapping used on the color sorting system is described in [12].

The color recognition method used for assigning a particular part face to a color class involves several steps. First, the 3-D histograms used to characterize each color class must be defined. The 3-D histogram for a particular class is calculated from a set of samples chosen to represent a desired color. A particular color class histogram represents the

0-7803-3932-0 
relative frequency of all colors found in the representative color samples. Second, a difference measurement is calculated to define the difference between the histograms of a reference color histogram and a particular panel part being sorted. The difference measurement is the $l_{l}$-norm or the sum of the absolute values of the difference between each color element in the two histograms. Finally, a threshold color difference value is calculated to assign parts to the most likely color class(es). For example, if the color difference between the histograms of a panel part and a reference color class exceeds the threshold value, the part will not be considered as belonging to that color class. Conversely, if the color difference is less than or equal to the threshold value, then the part is labeled as belonging to the color class. The initial threshold value is automatically computed such that classification errors are statistically minimized. However, initial thresholds can be manually adjusted for the desired level of classification performance. Complete details on the computation of the color class histograms, threshold values, and difference values are described in [8] and [9].

The actual sorting procedure for a particular panel part involves comparing its color histogram to those reference histograms of all defined color classes. The threshold values are used to acceptor reject the assignment of a part to each of the classes. Once the acceptance/rejection process is completed for each of the color classes, the panel part is assigned to the color class with the minimum color difference value among all acceptable color classes. If the part has been rejected for all color classes, it is then assigned to the out class. As mentioned earlier, the threshold values used to accept or reject parts in a particular class can be manually adjusted. Hence, in those applications where near perfect color sorts are required, the threshold can reduced. If, on the other hand, a larger variation of color is acceptable in the panels, the threshold can be increased. For instances where parts are designated as an out class, plant operators can be used to manually determine a part classification.

Once a color class has been assigned for each face of a panel part, a better face analysis is performed. The better face analysis requires that a priority of color classes be specified in advance. The color priority describes which color is most desirable or valuable. Given priority values, the better face analysis selects the part face with the highest priority. If the color of each face is of equal priority, then the better face is assigned to that which is closest to the reference histogram (smallest color difference).

Using the above color sorting methods require that the lighting conditions remain reasonably uniform over time. Hence, there is a need for continually monitoring color image quality such that variations in lighting are minimized during the color evaluation process. The method also requires that the lighting and the sensitivity of the CCD imaging elements be perfectly uniform across the field of view. Absolute lighting uniformity is impossible to achieve in an actual situation, but a shading correction algorithm [11] can be used to reduce the effects of inevitable variations.

The software system provides for three modes of operation: 1) real-time operation, 2) system training, and 3) system setup. Real-time operation involves those functions used in the actual sorting of parts and continually monitoring lighting variations. System training involves those functions used in specifying and characterizing different color classes. System training is based on showing the system a number of part faces that span the range of colors management allow in a given color class. Any number of color classes can be defined. However, the computational complexity goes up as the number of classes is increased. Also from a manufacturing viewpoint, more classes mean more space for sorting bins and more complicated material handling systems. Finally, system setup involves specifying different parameters, tolerances, and threshold values that an operator can use to control and fine tune the operation of the system.

\section{SYSTEM HARDWARE DESIGN}

For the color sorting system to be useful in an actual application, the system must have a throughput of at least 61 $\mathrm{cm}$ per second. The parts inspected by the system must be able to scan widths from $25 \mathrm{~mm}$ up to $190 \mathrm{~mm}$ and handle random lengths. Implementing the above color sorting algorithm along with other necessary image processing requirements such as color shading compensation and background extraction cannot easily be achieved to meet the real-time throughput requirements unless special purpose image processing and computing hardware are used.

Fig. 1 shows the overall system hardware design used to perform real-time color sorting operations. The system uses two Pulnix color line scan cameras to image the parts, one camera for each part face. Each color camera is connected to a color camera controller. The controllers govern the speed of the cameras and provide A/D conversion of the camera signal. The digital data coming out of each color camera controller is input to a special purpose image processing board that was designed and built at Virginia Tech. This board is called the Modular Reprogrammable Real-time Processing Hardware, or MORRPH [1], [3]. The MORRPH boards process the input camera signal and output the color measurement histograms from which parts are sorted into appropriate color classes. Consistent illumination is critical to the performance of the color sorting system. Hence, the system is designed to monitor the illumination levels and control them to within certain defined tolerances in real-time. 
Each color line scan camera is positioned so that its optical axis is perpendicular to the part face it is imaging (see Fig. 2). The parts run through the system on a belt driven material handling device. The optical axis is located $25 \mathrm{~mm}$ from this belt. Assuming that panel parts to be sorted will always exceed $25 \mathrm{~mm}$, this imaging geometry means the system never images a part edge but only a complete part face. The Pulnix line scan camera has 864 light sensitive elements. With a design image resolution of 2.5 pixels per $\mathrm{mm}$, the field of view of the Pulnix camera is sufficiently adequate to image part widths up to $190 \mathrm{~mm}$. The field of view of each camera is such that it not only images a part face but a white target as well. The white target is used to continually monitor variations in lighting.

The illumination system for the color sorting system must be as consistent and uniform as possible. The light sources used on the system employ tungsten halogen bulbs. These bulbs are used because the color temperature and light intensity does not vary much across bulb lifetime. Switching power supplies are used to provide conditioned and uniform power to the bulbs. Each switching power supply can accommodate an input signal that can be used to control the output voltage supplied to the light sources. This input allows the illumination control system to adjust the power supply voltage when lighting intensity falls outside a specified tolerance.

The MORRPH boards are the heart of the color sorting system. The MORRPHs perform most of the processing on the collected image data including shading compensation on the incoming color data, removing background pixels, mapping a 24-bit color image into a palette of 2,000 colors, computing the 3-D color histogram, and continuously monitoring the output of the light sources. The MORRPH ignores color camera data until it senses that a part has entered the field of view at which time it begins processing the image and computing the 3-D color histogram. The MORRPH stops computing color data when it senses that the part has left the field of view. The MORRPH continuously monitors the lighting, even when a part is not in the field of view. If the lighting changes beyond a defined tolerance, the MORRPH interrupts the image processing computer so that the computer can signal the light source power supply to increase or decrease the voltage supplied to the bulbs until the defined tolerance is regained.

After the MORRPH senses that a part has left the field of view of the camera, it immediately sends the color measurement data of the part face its camera has imaged to the image processing computer. This transfer occurs over the standard ISA bus. The image processing computer performs the pattern recognition algorithm discussed earlier to classify the color of each part face and perform a best face analysis.

\section{EVALUATION OF COLOR SORTING ACCURACY}

The accuracy of the color sorting algorithm was evaluated in an actual panel glue-up operation. A set of 25 color samples were selected to define each of six reference color classes for southern red-oak panel parts. These classes were (1) dark red, (2) red with some green, (3) dark brown, (4) medium brown, (5) light brown, and (6) red with some white. The selected samples were chosen by experienced mill operators such that any combination of the 25 parts within a class would result in a clear panel. The 25 samples for each of the six classes were then used to train the color sorting system. The end result of the training procedure resulted in six reference 3-D color histograms that represented each of the color classes. Light uniformity during the time of training was held constant.

After training the system, the performance of the part sorting algorithm was tested. This particular glue-up operation graded edge-glued panels into three categories: 1) clear, 2) acceptable, and 3) unacceptable. Clear panels have approximately the same color across the better face and are the most valuable panels. Acceptable panels have color characteristics that are within acceptable bounds but have some allowable color variation that can be compensated with darker finishes. Unacceptable panels have color characteristics which vary widely across their better face and are typically used to create painted panels. This particular manufacturing operation has set a production goal to generate at least 90 percent clear and acceptable panels.

The in-plant tests involved determining the percentage of clear and acceptable panels created from 17 randomly selected pallet loads of parts. One load typically contains 900-1000 pieces. Each of these pieces were color sorted with the color sorting system and then glued up into panels required for the production run. The average rate of clear and acceptable panels for the entire experiment was 91.3 percent. The highest and lowest rates achieved were 99.1 and 83.0 percent, respectively. The lowest rates in the study were attributed to miss-calibrated light levels, dust, and parts that had significantly different color variations that were not represented by any of the six color classes used to train the system. Overall, these results indicate that the system is capable of creating high-quality edge-glued panels. However, proper color class training and maintenance of uniform lighting is critical for accurate results. Proper training requires a good understanding of the total color variability in wood, how to segment the total variability into representative 
classes, and how changing light standards can affect the performance of the system.

\section{SUMMARY}

This paper has described a color sorting system for use in sorting edge-glued panel parts. Operational in-plant tests indicate that the system performs very well for color sorting red-oak panel parts. The system can be trained for other wood species as well. The introduction of the system into the plant environment was well received by plant employees. The system is now commercially available and is currently being marketed by a forest products equipment manufacturer. Fig. 3 shows a commercialized version of the Virginia Tech color sorting system.

\section{REFERENCES}

[1]C. Brunner, B. Shaw, D. A. Butler, and J. W. Funck, "Using color in machine vision systems for wood processing," Wood and Fiber Science, vol. 22, no. 4, 1990, pp. 413-428.

[2]R. Conners, C. McMillin, and C. Ng, "The utility of color information in the location and identification of defects in surfaced hardwood lumber," Proceedings of the First International Conference on Scanning Technology in Sawmilling, San Francisco, California, October 10-11, 1983.

[3]T. Drayer, W. King, J. Tront, and R. Conners, "A modular and reprogrammable real-time processing hardware, MORRPH," Proceedings of the IEEE Symposium on FPGAs for Custom Computing Machines, Napa Valley, California, April 19-21, 1995.

[4]T. Drayer, W. King, J. Tront, and R. Conners, "Using multiple FPGA architectures for real-time processing of low-level machine vision functions," Proceedings of IECON '95, 1995.

[5]R. Duda, and P. Hart, Pattern Classification and Scene Analysis, John Wiley and Sons, New York, 1973.

[6]L. Haney, C. Precetti, H. Gibson, G. Krutz, and D. Cassens, "Color matching of weed with a real-time machine vision system," American Society of Agricultural Engineers, Atlanta, Georgia, December 13-16, 1994.

[7]P. Heckberg "Color image quantitization for frame buffer display," Computer Graphics, Vol. 16, No. 3, 1983, pp. 297-307.
[8]Q. Lu, "A real-time system for color-sorting edge-glued panel parts," Master's Thesis in preparation, Department of Electrical Engineering, Virginia Tech, Blacksburg, Virginia.

[9]Q. Lu, R. Conners and D. Kline, "A real-time algorithm for color sorting edge-glued panel parts," International Conference on Image Processing, Santa Barbara, CA, Oct 26-29, 1997.

[10]A. Pugel, J. Phelps, and D. Stokke, "Improving visual color sorting of dimension stock," Forest Products Journal, Vol. 45 No. 11/12, 1995, pp. 57-60.

[11]A. Sawchuk, "Real-time correction of intensity nonlinearities in imaging system," IEEE Transactions on Computers, Vol. 26, No. 1, 1977, pp. 34-39.

[12]S. Srikanteswara, Q. Lu, W. King, T. Drayer, R Conners, D. Kline, and P. Araman, "Real-time implementation of a color sorting system," Technical Conference on Machine Vision Applications, Architectures, and Systems Integration VI, Pittsburg, PA, 14-17 October 1997.

[13]S. Yoo, C. Precetti, and D. Cassens, "Color machine vision used to establish color grading standards for hardwood dimension parts," American Society of Agricultural Engineers, Nashville, Tennessee, December 15-18, 1992. 


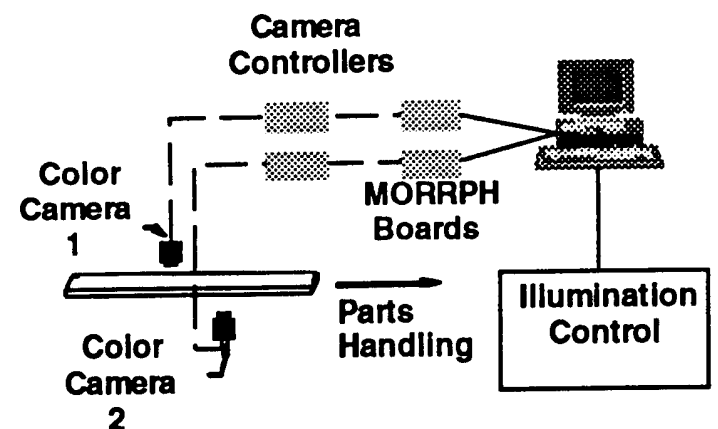

Fig. 1. Overall system hardware design for the color sorting system

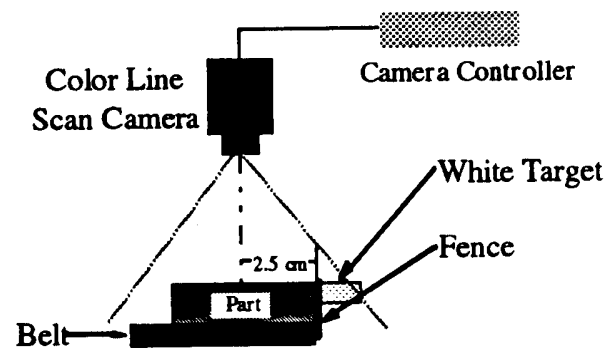

Fig. 2. Color imaging geometry.

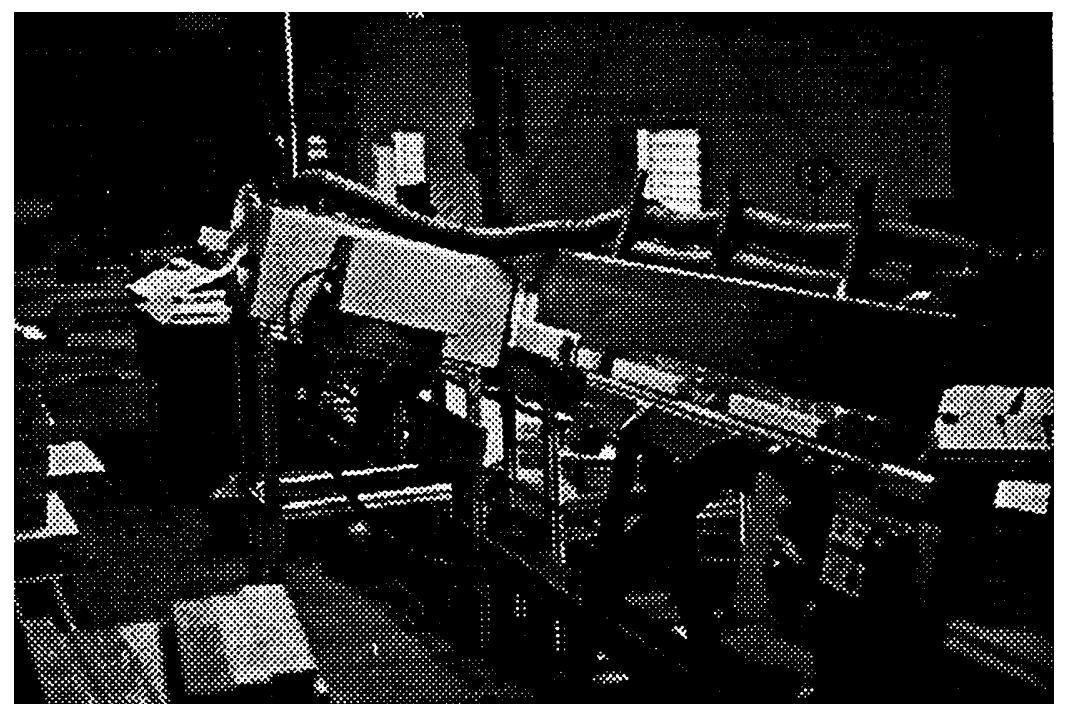

Fig. 3. Commercialized version of the Virginia Tech color sorting system. 


\title{
Proceedings of the
}

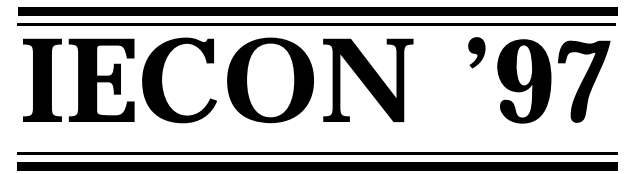

\section{3rd International Conference on Industrial Electronics, Control, and Instrumentation}

\author{
Volume 3 of 4 \\ Emerging Technologies \\ Factory Automation \\ Robotics, Vision, and Sensors
}
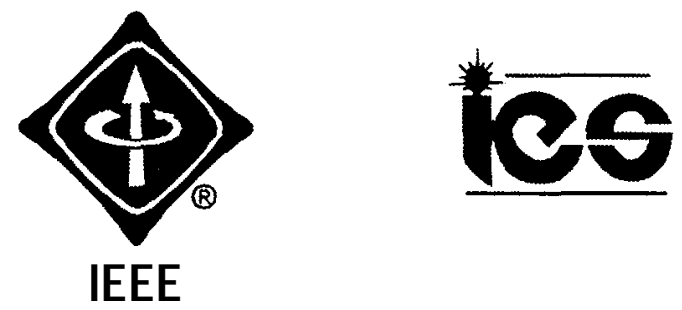

Sponsored by:

IEEE Industrial Electronics Society

Technical Co-Sponsor:

Society of Instrument and Control Engineers of Japan (SICE) 